\title{
Adaptations of the Early Embryonic Heart to Increase Hemodynamic Load
}

\author{
Sandra Rugonyi
}

Biomedical Engineering, Oregon Health \& Science University, Portland, OR, USA

Heart development is the result of intrinsic genetic programs, which are modulated by biochemical and hemodynamic conditions during gestation. Normal blood flow is essential for proper cardiac development, and altered blood flow through the heart at early embryonic stages leads to congenital heart malformations [1]. The developing heart is continuously adapting to changes in blood flow that occur as a result of embryonic growth. Details of cardiac adaptations to changes in blood flow conditions that take place during early embryonic stages, however, are only recently starting to emerge. This is in great part thanks to advances in imaging technologies that are enabling more detailed characterization of mechanical and biological changes that occur in the tiny embryonic heart when blood flow conditions are altered.

During early developmental stages, the vertebrate heart is a linear tube that soon bends and twists forming a looping tubular heart. Following looping, the heart chambers and valves begin to form. Looping stages are very sensitive to hemodynamic conditions, and cardiac adaptations during this period have repercussions during valve and chamber formation stages. The tubular heart consists of consecutive cardiac segments: the atrium, the ventricle and the outflow tract (OFT). The OFT is a critical cardiac segment, as it gives rise to the intraventricular septum, pulmonary and aortic outlets, and their valves, and is the site of origin for a large proportion of cardiac defects. To better understand how cardiac defects arise, we are focusing on early embryonic adaptations of OFT tissues to altered hemodynamic conditions.

Using chicken embryos as animal models of cardiovascular development, we are employing a combination of in vivo imaging and physiological measurements, immunohistochemistry, and computational techniques to elucidate how the embryonic heart adapts to changes in hemodynamic conditions. In chick embryos, cardiac beating starts at Hamburger-Hamilton (HH) stage 10 (33-38 hrs of incubation), and septation occurs after HH24 (day 4). We alter hemodynamic conditions in the heart at HH18 (day 3) through OFT banding, a procedure in which a surgical suture is passed around the heart OFT and tightened to constrict the lumen cross-sectional area.

To monitor changes in hemodynamic conditions immediately after banding (within 2 hrs), we use optical coherence tomography (OCT) to image the beating heart, and we employ a micro-pressure servonull system to measure blood pressure. OCT images and pressure data are acquired simultaneously with electrocardiogram (ECG) signals, which allow synchronization of events to the cardiac cycle. These data enable detailed analysis of hemodynamic changes after banding.

To determine cardiac tissue adaptations to changes in hemodynamic conditions after 24 hrs of banding, that is at HH24 (day 4), we use immunohistochemistry labeling of key components of the cardiac tissue 
extracellular matrix (collagens I, III, VI and XIV) followed by imaging with confocal microscopy. Reconstruction of images in 3D allows visualization of localized changes in the composition of the OFT tissues in response to banding.

We found that both hemodynamic conditions and the degree of tissue adaptation in the OFT depend on band tightness. We obtained the band tightness (or degree of band constriction) by measuring the OFT diameter before and after banding using OCT, and by measuring the band diameter from confocal images. We found that blood pressure and blood pressure amplitudes increased after banding in the heart ventricle, aortic sac (the outlet of the OFT) and dorsal aorta (arterial circulation) of the chick embryo, with increased tightness resulting in more elevated pressures. Further, the pulse transit time in the OFT, the time it takes the pressure wave to travel through the OFT, significantly decreased after banding, from about $10 \%$ of the cardiac cycle for normal and control embryos, to almost zero for band tightness $>35 \%$. Thus banding has a significant effect not only on blood pressure, but also on the speed of the pressure wave through the OFT. This later result was confirmed with OCT imaging.

After $24 \mathrm{hrs}$ of banding, deposition of collagens I and III in the OFT was not significantly affected (as compared to control embryos), but deposition of collagens VI and XIV increased significantly, and correlated with band tightness. Collagen XIV increased deposition both distally and proximally. Distal to the band, collagen VI deposition was elevated in the OFT outer curvature. In the proximal OFT, however, deposition was increased only in endocardial cells, which line the cardiac tissue and are in contact with the OFT lumen. Thus deposition of collagens VI and XIV was not only increased, but also localized within the OFT.

Our results indicate that banding leads to increased blood pressure, but also increased pressure pulse wave velocities. These changes affect both the distribution and gradients of strains in OFT tissues, which likely affect adaptation mechanisms that rely on mechanotransduction. The increase in collagen VI and XIV deposition in banded hearts, suggests that these collagens play a key role in structural adaptation to increased loading at early stages of heart development, while the localized patterns of deposition likely reflect local shear and wall strain patterns in the OFT. The dependence of both collagen deposition and hemodynamic changes on band tightness indicates a strong role of mechanotransduction mechanisms in the embryonic adaptation of the heart to hemodynamic conditions. In the future this data will certainly lead to a better understanding of embryonic cardiovascular adaptations to blood flow conditions, and how these adaptations can lead to congenital heart defects [2].

\section{References}

[1] Goenezen, S., M. Rennie, and S. Rugonyi, Biomechanics and Modeling in Mechanobiology, 11 (2012), p. 1187-1204.

[2] The author acknowledges funding from NIH (R01 HL094570) and NSF (DBI-1052688). Further, the author acknowledges the contributions of Dr. Rennie, Dr. Shi and Dr. Liu in the development of this study: without their hard work, this study would not have been possible. 